\begin{tabular}{|c|l|}
\hline Title & Remarks on proofs of conservation laws for nonlinear Schrödinger equations \\
\hline Author(s) & Ozawa, Tohru \\
\hline Citation & Hokkaido University Preprint Series in Mathematics, 706, 1-6 \\
\hline Issue Date & 2005 \\
\hline DOI & 10.14943/83857 \\
\hline Doc URL & http://hdl.handle.net/2115/69511 \\
\hline Type & bulletin (article) \\
\hline File Information & pre706.pdf \\
\hline
\end{tabular}

Instructions for use 


\title{
Remarks on proofs of conservation laws for nonlinear Schrödinger equations
}

Dedicated to Professor Nakao Hayashi on his fiftieth birthday

\author{
T. Ozawa
}

Department of Mathematics, Hokkaido University, Sapporo 060-0810, Japan

\begin{abstract}
Conservation laws of the charge and of the energy are proved for nonlinear Schrödinger equations with nonlinearities of gauge invariance in a way independent of approximate solutions.
\end{abstract}

\section{Introduction}

In this paper we consider the Cauchy problem for nonlinear Schödinger equations of the form

$$
i \partial_{t} u+\frac{1}{2} \Delta u=f(u)
$$

where $u$ is a complex-valued function of $(t, x) \in \mathbb{R} \times \mathbb{R}^{n}, \partial_{t}=\partial / \partial t, \Delta$ is the Laplacian in $\mathbb{R}^{n}$, and $f(u)$ is a nonlinear interaction given by a complex-valued function $f$ on $\mathbb{C}$.

There is a large literature on the Cauchy problem for (NLS) (see for instance $[1,2,6]$ and references therein). The standard treatment proceeds in two steps. Frist, by a contraction argument we prove the existence and uniqueness of local solutions to the integral equations associated with (NLS) with prescribed Cauchy data. Local solutions on the time intervals of the form $[-T, T]$ with $T>0$ are given as fixed points of the integral equations as contraction mappings on closed balls in function spaces on $[-T, T]$. Basic tools here are provided by the Strichartz estimates. The corresponding contraction factor in that argument can be smaller than one by taking $T$ sufficiently small. The next step is to extend the local solutions beyond the time interval $[-T, T]$ on the basis of a priori estimates of the solutions, given by conservation laws. Conservation laws of the charge and of the energy are of particular interest via direct relations to $L^{2}$ and $H^{1}$ 
solutions, respectively. According to $[2,5]$, we specify the assumptions on nonlinearities as follows.

$$
f \in C^{1}(\mathbb{C} ; \mathbb{C}), f(0)=0 \text {, and for some } p \text { with } 1<p<\infty \quad f \text { satisfies the }
$$

estimate

$$
\left|f^{\prime}(z)\right| \leq C\left(1+|z|^{p-1}\right)
$$

for all $z \in \mathbb{C}$, where $\left|f^{\prime}(z)\right|=\max \left(\left|\frac{\partial f}{\partial z}\right|,\left|\frac{\partial f}{\partial \bar{z}}\right|\right)$.

(H2) $\operatorname{Im}(\bar{z} f(z))=0$ for all $z \in \mathbb{C}$.

(H3) There exists $V \in C^{1}(\mathbb{C} ; \mathbb{R})$ such that $V(0)=0$ and $f(z)=\partial V / \partial \bar{z}$.

We denote by $(\cdot, \cdot)$ the scalar product in $L^{2}$ as well as its extension to a duality coupling on $B \times B^{\prime}$, where $B$ is a Banach space such that $B \hookrightarrow L^{2} \hookrightarrow B^{\prime}$ with dense embeddings. Under the assumption (H2), a formal proof of the conservation law of the charge is given by taking the real part of the scalar product between $i u$ and (NLS) as follows:

$$
\begin{aligned}
0 & =2 \operatorname{Re}\left(i \partial_{t} u+\frac{1}{2} \Delta u-f(u), i u\right) \\
& =2 \operatorname{Re}\left(\partial_{t} u, u\right)+\operatorname{Im}(\Delta u, u)-2 \operatorname{Im}(f(u), u)=\frac{d}{d t}\|u\|_{2}^{2}
\end{aligned}
$$

For an $L^{2}$-solution, (1.1) does not make sense since $\left(\partial_{t} u, u\right)$ and $(\Delta u, u)$ do not make sense. To justify (1.1) in a duality argument, we require that $u$ is at least an $H^{1}$-solution.

Under the assumption (H3), a formal proof of the conservation law of the energy is given by taking the real part of the scalar product between $-\partial_{t} u$ and (NLS) as follows:

$$
\begin{aligned}
0 & =2 \operatorname{Re}\left(i \partial_{t} u+\frac{1}{2} \Delta u-f(u),-\partial_{t} u\right) \\
& =-\operatorname{Re}\left(\Delta u, \partial_{t} u\right)+2 \operatorname{Re}\left(f(u), \partial_{t} u\right)=\frac{d}{d t} E(u),
\end{aligned}
$$

where

$$
E(u)=\frac{1}{2}\|\nabla u\|_{2}^{2}+\int_{\mathbb{R}^{n}} V(u) d x .
$$

For an $H^{1}$-solution, (1.2) does not make sense since $\left(\partial_{t} u, \partial_{t} u\right)$ and $\left(\partial_{t} u, \Delta u\right)$ do not make sense. To justify (1.2), we require that $u$ is at least an $H^{2}$-solution.

There is a natural question how one can prove those conservation laws in a framework of regularity of solutions where everything makes sense. As is pointed out by Ginibre [2], to appreciate the difficulty of the question, we may think of the uniqueness of solutions constructed by compactness methods for NLS with defocusing nonlinearities of supercritical Sobolev exponents (see also Remark 9.4.7 in [1]).

There are basically two methods of proofs for conservation laws of the charge and of the energy. One is based on the continuous dependence of solutions on the Cauchy data, by which $H^{j}$-solutions are approximated by a sequence of $H^{j+1}$-solutions for $j=0,1$, so that formal computations such as (1.1) and (1.2) are justified for approximate solutions and actual conserved quantities are realized in a limiting procedure $[4,5]$. The other is 
based on a sequence of regularized equations which are compatible with methods for local resolution and with formal computations such as (1.1) and (1.2) [3, 7]. Note that both methods involve a limiting procedure on approximate solutions.

The purpose in this paper is to prove conservation laws of the charge and of the energy in a way essentially independent of approximating procedure.

Instead, we exploit additional properties of solutions provided by the Strichartz estimates. To be specific, the Strichartz estimates are formulated in the following lemma (see $[1,2,5,6]$ for instance).

Definition. A pair of two exponents $(q, r)$ is called an admissible pair if and only if $0 \leq 2 / q=n / 2-n / r \leq 1$ with the exception $(n, q, r)=(2,2, \infty)$.

Lemma. Let $(q, r),\left(q_{j}, r_{j}\right), j=1,2$, be admissible pairs. Then the free propagator $U(t)=\exp (i(t / 2) \Delta)$ satisfies the following estimates.

$$
\begin{aligned}
& \left\|U(t) \phi ; L^{q}\left(\mathbb{R} ; L^{r}\left(\mathbb{R}^{n}\right)\right)\right\| \leq C_{r}\left\|\phi ; L^{2}\left(\mathbb{R}^{n}\right)\right\|, \\
& \left\|(G v)(t) ; L^{q_{1}}\left(I ; L^{r_{1}}\left(\mathbb{R}^{n}\right)\right)\right\| \leq C_{r_{1}} C_{r_{2}}\left\|v ; L^{q_{2}^{\prime}}\left(I ; L^{r_{2}^{\prime}}\left(\mathbb{R}^{n}\right)\right)\right\|,
\end{aligned}
$$

where $G$ is the integral operator defined by

$$
(G v)(t)=\int_{0}^{t} U\left(t-t^{\prime}\right) v\left(t^{\prime}\right) d t^{\prime}
$$

$I \subset \mathbb{R}$ is an interval with $0 \in \bar{I}, C_{r}$ is a constant independent of $I$, and $q^{\prime}$ is the dual exponent defined by $1 / q+1 / q^{\prime}=1$.

The main results in this paper are as follows.

Proposition 1. Let $f$ satisfy (H1) and (H2). Let $\phi \in L^{2}$. Let $T>0$ and let $u$ be a solution of the integral equation

$$
u(t)=U(t) \phi-i \int_{0}^{t} U\left(t-t^{\prime}\right) f\left(u\left(t^{\prime}\right)\right) d t^{\prime}
$$

with $u \in L^{q}\left(-T, T ; L^{r}\right)$ for some admissible pair $(q, r)$. Then $\|u(t)\|_{2}=\|\phi\|_{2}$ for all $t \in[-T, T]$.

Proposition 2. Let $f$ satisfy (H1) and (H3). Let $\phi \in H^{1}$. Let $T>0$ and let $u$ be a solution of (1.4) with $u \in L^{q}\left(-T, T ; H_{r}^{1}\right)$ for some admissible pair $(q, r)$. Then $E(u(t))=E(\phi)$ for all $t \in[-T, T]$, where $E(u)$ is as in (1.3).

Remarks 1. Concerning $L^{2}$ solutions, it is known that if $u$ is a solution of (1.4) with $u \in L_{t}^{q} L^{r}$ for some admissible pair $(q, r)$, then $u \in C_{t}\left(L^{2}\right) \cap L_{t}^{q} L^{r}$ for all admissible pair $(q, r)$. A similar result holds for $H^{1}$ solutions. See [1] for instance. 
Remarks 2. Typical examples of admissible pairs are

$$
(q, r)=(\infty, 2) \text { and }(q, r)=(4(p+1) /(n(p-1)), p+1)
$$

The existence and uniqueness of local $L^{2}\left[\right.$ resp. $\left.H^{1}\right]$ solutions of (1.4) is well-known if $p \leq 1+4 / n$ [resp. $(n-2) p \leq n+2]$. See $[1,2,5,6]$. In the next section we prove Propositions 1 and 2 in a way independent of approximate solutions. The proofs depend on the integral representation (1.4) of solutions and on regularity and integrability properties of local solutions given by the Strichartz estimates.

\section{Proofs of the propositions}

Proof of Proposition 1. We rewrite (1.4) as

$$
U(-t) u(t)=\phi-i \int_{0}^{t} U\left(-t^{\prime}\right) f\left(u\left(t^{\prime}\right)\right) d t^{\prime}
$$

By the unitarity of the free propagator, we have

$$
\begin{aligned}
& \|u(t)\|_{2}^{2} \\
& =\|U(-t) u(t)\|_{2}^{2} \\
& =\|\phi\|_{2}^{2}-2 \operatorname{Im}\left(\phi, \int_{0}^{t} U\left(-t^{\prime}\right) f\left(u\left(t^{\prime}\right)\right) d t^{\prime}\right)+\left\|\int_{0}^{t} U\left(-t^{\prime}\right) f\left(u\left(t^{\prime}\right)\right) d t^{\prime}\right\|_{2}^{2} .
\end{aligned}
$$

The middle term on the RHS of (2.2) is equal to

$$
-2 \operatorname{Im} \int_{0}^{t}\left(U\left(t^{\prime}\right) \phi, f\left(u\left(t^{\prime}\right)\right)\right) d t^{\prime},
$$

where the time integral of the scalar product is understood to be the duality coupling on $\left(L_{t}^{\infty} L^{2} \cap L_{t}^{q} L^{p+1}\right) \times\left(L_{t}^{1} L^{2}+L_{t}^{q^{\prime}} L^{(p+1) / p}\right)$ with $q=4(p+1) /(n(p-1))$, while the last term on the RHS of (2.2) is equal to

$$
\begin{aligned}
& 2 \operatorname{Re} \int_{0}^{t}\left(f\left(u\left(t^{\prime}\right)\right), \int_{0}^{t^{\prime}} U\left(t^{\prime}-t^{\prime \prime}\right) f\left(u\left(t^{\prime \prime}\right)\right) d t^{\prime \prime}\right) d t^{\prime} \\
& =-2 \operatorname{Im} \int_{0}^{t}\left(f\left(u\left(t^{\prime}\right)\right), u\left(t^{\prime}\right)+i \int_{0}^{t^{\prime}} U\left(t-t^{\prime \prime}\right) f\left(u\left(t^{\prime \prime}\right)\right) d t^{\prime \prime}\right) d t^{\prime} \\
& =-2 \operatorname{Im} \int_{0}^{t}\left(f\left(u\left(t^{\prime}\right)\right), U\left(t^{\prime}\right) \phi\right) d t^{\prime} \\
& =2 \operatorname{Im} \int_{0}^{t}\left(U\left(t^{\prime}\right) \phi, f\left(u\left(t^{\prime}\right)\right)\right) d t^{\prime},
\end{aligned}
$$

where we have used (H2) and (1.4) in the first and second equalities, respectively. We have thus proved that the middle and last terms on the RHS of (2.2) cancel out. This completes the proof. 
Proof of Proposition 2. In a way similar to the preceding argument, we compute

$$
\begin{aligned}
& \|\nabla u(t)\|_{2}^{2} \\
& =\|\nabla U(-t) u(t)\|_{2}^{2} \\
& =\left\|\nabla \phi-i \int_{0}^{t} U\left(-t^{\prime}\right) \nabla\left(f\left(u\left(t^{\prime}\right)\right)\right) d t^{\prime}\right\|_{2}^{2} \\
& =\|\nabla \phi\|_{2}^{2}-2 \operatorname{Im}\left(\nabla \phi, \int_{0}^{t} U\left(-t^{\prime}\right) \nabla\left(f\left(u\left(t^{\prime}\right)\right)\right) d t^{\prime}\right) \\
& +\left\|\int_{0}^{t} U\left(-t^{\prime}\right) \nabla\left(f\left(u\left(t^{\prime}\right)\right)\right) d t^{\prime}\right\|_{2}^{2} \\
& =\|\nabla \phi\|_{2}^{2}-2 \operatorname{Im} \int_{0}^{t}\left(U\left(t^{\prime}\right) \nabla \phi, \nabla\left(f\left(u\left(t^{\prime}\right)\right)\right)\right) d t^{\prime} \\
& +2 \operatorname{Re} \int_{0}^{t}\left(\nabla\left(f\left(u\left(t^{\prime}\right)\right)\right), \int_{0}^{t^{\prime}} U\left(t^{\prime}-t^{\prime \prime}\right) \nabla\left(f\left(u\left(t^{\prime \prime}\right)\right)\right) d t^{\prime \prime}\right) d t^{\prime} \\
& =\|\nabla \phi\|_{2}^{2}+2 \operatorname{Im} \int_{0}^{t}\left(\nabla\left(f\left(u\left(t^{\prime}\right)\right), U\left(t^{\prime}\right) \nabla \phi\right) d t^{\prime}\right. \\
& +2 \operatorname{Im} \int_{0}^{t}\left(\nabla\left(f\left(u\left(t^{\prime}\right)\right)\right),-i \int_{0}^{t^{\prime}} U\left(t^{\prime}-t^{\prime \prime}\right) \nabla\left(f\left(u\left(t^{\prime \prime}\right)\right)\right) d t^{\prime \prime}\right) d t^{\prime} \\
& =\|\nabla \phi\|_{2}^{2}+2 \operatorname{Im} \int_{0}^{t}\left(\nabla\left(f\left(u\left(t^{\prime}\right)\right)\right), \nabla u\left(t^{\prime}\right)\right) d t^{\prime} \\
& =\|\nabla \phi\|_{2}^{2}-2 \operatorname{Im} \int_{0}^{t}\left(f\left(u\left(t^{\prime}\right)\right), \Delta u\left(t^{\prime}\right)\right) d t^{\prime} \\
& =\|\nabla \phi\|_{2}^{2}-4 \operatorname{Re} \int_{0}^{t}\left(f\left(u\left(t^{\prime}\right)\right), \partial_{t} u\left(t^{\prime}\right)\right) d t^{\prime} \\
& =\|\nabla \phi\|_{2}^{2}-2 \int_{0}^{t} \frac{d}{d t} \int_{\mathbb{R}^{n}} V\left(u\left(t^{\prime}\right)\right) d x d t^{\prime} \\
& =\|\nabla \phi\|_{2}^{2}-2 \int_{\mathbb{R}^{n}} V(u(t)) d x+2 \int_{\mathbb{R}^{n}} V(\phi) d x,
\end{aligned}
$$

where the last two time integrals of the scalar products are understood to be the duality coupling on $\left(L_{t}^{1} H^{1}+L_{t}^{q^{\prime}} H_{(p+1) / p}^{1}\right) \times\left(L_{t}^{\infty} H^{1} \cap L_{t}^{q} H_{p+1}^{1}\right)$ with $q=4(p+1) /(n(p-1))$, and

$$
\begin{aligned}
& \operatorname{Im}(f(u), \Delta u)=\lim _{\varepsilon \downarrow 0} \operatorname{Im}\left((1-\varepsilon \Delta)^{-1} f(u),(1-\varepsilon \Delta)^{-1} \Delta u\right) \\
& =\lim _{\varepsilon \downarrow 0} \operatorname{Im}\left((1-\varepsilon \Delta)^{-1} f(u),(1-\varepsilon \Delta)^{-1}\left(-2 i \partial_{t} u+2 f(u)\right)\right) \\
& =\lim _{\varepsilon \downarrow 0} \operatorname{Im}\left((1-\varepsilon \Delta)^{-1} f(u),(1-\varepsilon \Delta)^{-1}\left(-2 i \partial_{t} u\right)\right) \\
& =2 \operatorname{Re}\left(f(u), \partial_{t} u\right) .
\end{aligned}
$$

This completes the proof.

Acknowledgments. The author is grateful to the referee for useful suggestions. 


\section{References}

[1] T. Cazenave, "Semilinear Schrödinger Equations", Courant Lecture Notes in Mathematics, Vol. 10, American Mathematical Society, 2003.

[2] J. Ginibre, An introduction to nonlinear Schrödinger equations, in "Nonlinear Waves" (R. Agemi, Y. Giga and T. Ozawa, Eds.), GAKUTO International Series, Mathematical Sciences and Applications, Vol.10, Gakkōtosho, Tokyo, 1997.

[3] J. Ginibre and G. Velo, On a class of nonlinear Schrödinger equations, I. The Cauchy problem, J. Funct. Anal. 32 (1979), 1-32.

[4] T. Kato, On nonlinear Schrödinger equations, Ann. Inst. H. Poincaré, Phys. Théor. 46 (1987), 113-129.

[5] T. Kato, Nonlinear Schrödinger equations, in "Schrödinger Operators" (H. Holden and A. Jensen, Eds.), Lecture Notes in Phys. 345, Springer, 1989.

[6] C. Sulem and P. L. Sulem, "The Nonlinear Schrödinger Equation: Self-Focusing and Wave Collapse," Applied Mathematical Sciences, Vol. 139, Springer, 1999.

[7] Y. Tsutsumi, $L^{2}$-solutions for nonlinear Schrödinger equations and nonlinear groups, Funkcialaj Ekvacioj 30 (1987), 115-125. 